\title{
Estudo comparativo de três aparelhos foraminais eletrônicos na determinação do comprimento de trabalho
}

\section{Comparative study of three foraminal electronic devices to determine working length}

\author{
José Umberto BAMPA ${ }^{1}$, Sonia Maria Vidolin Junqueira FRANCO ${ }^{1}$, Ana Emília Farias PONTES ${ }^{1}$, \\ Devanir de Araújo CERVI ${ }^{1}$ \\ ${ }^{1}$ Curso de Odontologia, Centro Universitário da Fundação Educacional de Barretos (UNIFEB) - Barretos \\ (SP), Brasil.
}

\begin{abstract}
Resumo
A terapia endodôntica depende de várias etapas. Para se alcançar sucesso é necessário que todas as etapas sejam bem realizadas, sendo a odontometria uma delas. Por isso é importante que se busque o aprimoramento das técnicas e materiais. O comprimento de trabalho (CRT) correto fará com que a lima exerça a instrumentação até à constrição exata entre o canal dentinário e o canal cementário, sem que ocorra agressão aos tecidos periapicais. O objetivo deste estudo foi comparar in vivo e in vitro três marcas de localizadores apicais quanto a sua eficácia na determinação do comprimento de trabalho durante tratamento de canal radicular. Oito dentes maxilares com indicação para exodontia foram utilizados neste trabalho. Depois das mensurações in vivo com os localizadores apicais, as amostras foram extraídas e realizados os procedimentos in vitro com limas manuais tipo Kerr. Como resultado deste estudo, a maior amplitude de variação foi do aparelho Just II (4,0 mm, variando de $-1,5 \mathrm{~mm}$ a 2,5 mm). A menor foi do Mini Root ZX (2,5 mm, variando de $-1,0 \mathrm{~mm}$ a 1,5 mm). O aparelho Propex II teve valor intermediário $(3,5 \mathrm{~mm}$, variando de $-0,5 \mathrm{~mm}$ a 3,0 $\mathrm{mm}$ ). Pode-se concluir que os aparelhos testados mostraram eficácias similares na mensuração do CRT in vitro e in vivo. O aparelho Root ZX-II mostrou tendência de melhor desempenho.
\end{abstract}

Palavras-chave: endodontia; odontometria; equipamentos odontológicos.

\begin{abstract}
Endodontic therapy is dependent on several steps. To be successful, all of its steps should be well performed, including odontometry. Therefore, it is important to seek improvement of techniques and materials. Correct working length (CWL) allows the file to perform instrumentation to the exact constriction between the dentinal canal and the cementery canal, without aggression to periapical tissues. The objective of this study was to compare, in vivo and in vitro, three brands of apical locators with respect to their effectiveness in determining the working length during root canal treatment. Eight maxillary teeth indicated for extraction were used in this study. After in vivo measurements using the apical locators, the samples were extracted and the in vitro procedures were performed using Kerr type hand files. The results obtained with the three locators were as follows: Just II presented the largest variation amplitude (4.0 mm, ranging from $-1.5 \mathrm{~mm}$ to $2.5 \mathrm{~mm})$; Mini Root $Z X$ showed the shortest variation $(2.5 \mathrm{~mm}$, ranging from $-1.0 \mathrm{~mm}$ to $1.5 \mathrm{~mm})$; and Propex II presented an intermediate value $(3.5 \mathrm{~mm}$, ranging from $0.5 \mathrm{~mm}$ to $3.0 \mathrm{~mm})$. In conclusion, the three devices tested show similar performance in measuring CWL in vitro and in vivo, and the best results were achieved using Mini Root $Z X$.
\end{abstract}

Keywords: endodontic; odontometry; dental equipment.

Autor para correspondência: José Umberto Bampa - Centro Universitário da Fundação Educacional de Barretos (UNIFEB) - Avenida Prof. Roberto Frade Monte, 389 - Aeroporto - CEP: 14783-226 -

Barretos (SP), Brasil - E-mail: clinicabampa@terra.com.br

Recebido em: 31 de março de 2014

Aceito para publicação em: 27 de outubro de 2015

http://dx.doi.org/10.4322/1980-0029.172014 


\section{Introdução}

Os procedimentos envolvidos no tratamento endodôntico devem se limitar ao canal dentário principalmente no limite cemento-dentina-canal, pois esta estrutura localizada no interior do canal radicular não pode ser clinicamente identificada (LISKA, 2009). Neste sentido, um dos principais desafios seria determinar, com precisão, o comprimento real de trabalho para se obter uma adequada instrumentação e obturação (LUCISANO et al., 2009; OROSCO, 2010).

De acordo com Kuttler (1955), o limite apical ideal para instrumentação e obturação dos canais radiculares localiza-se na junção cemento-dentina-canal, cuja localização é variável. A determinação de um correto comprimento de trabalho é um ponto importante para o sucesso da terapia endodôntica (CESÁRIO et al., 2014). Erros na odontometria, por descuido ou imperícia, podem resultar em dor pós-operatória, perfurações apicais, sub ou sobre-instrumentações, além de instrumentação e obturação deficientes e incompletas, levando ao insucesso da terapia endodôntica (FREITAS et al., 2012).

Vários métodos e técnicas já foram propostos para determinação do comprimento de trabalho do canal radicular. Desde a resposta do paciente à dor, provocada pela ultrapassagem de um instrumento pelo forame apical até o emprego de radiografias convencionais e digitais (BRITO JUNIOR et al., 2007). Dentre elas, o exame radiográfico tem sido o mais popular. Vários autores demonstram que o comprimento do canal determinado, radiograficamente, varia consideravelmente em relação ao comprimento real do canal (MATTAR e ALMEIDA, 2008). Devido às dificuldades encontradas com o método radiográfico, os localizadores apicais de última geração se apresentam como recurso importante para determinar a odontometria de forma mais segura (GORDON e GHANDLER, 2004).

Um avanço tecnológico adveio do desenvolvimento de aparelhos para detecção eletrônica da constrição apical, obtendo em diversos estudos alta taxa de confiabilidade, aparelhos estes chamados de localizadores apicais eletrônicos (SIU et al., 2009). Sua funcionalidade está baseada no fato de a condutividade elétrica dos tecidos ao redor do ápice radicular ser maior que a verificada no interior do sistema de canais radiculares, em condição úmida ou seca, ou mesmo preenchido com líquido não condutivo (SANTANA, 2007).
Nos últimos anos, a determinação eletrônica do comprimento de trabalho ganhou popularidade tanto entre os clínicos gerais quanto entre os endodontistas, principalmente porque se comprovou que sua utilização como coadjuvante no preparo biomecânico reduz o número de radiografias necessárias para a determinação do limite apical de instrumentação e obturação (MIGUITA et al., 2011).

O objetivo deste trabalho foi avaliar a eficácia clínica de três localizadores apicais aplicados in vivo e in vitro para a mensuração dos canais radiculares.

\section{Material e Métodos}

O presente estudo foi aprovado pelo Comitê de Ética em Pesquisa do Centro Universitário da Fundação Educacional de Barretos (UNIFEB), sob o Parecer- $n^{\circ} 503.678$ de 3/9/2013.

As amostras deste trabalho foram compostas por 4 dentes molares superiores (dentes 17, 18, 26 e 28), dois segundos pré-molares superiores (15 com duplo canal e dente 25 com raiz única), 1 incisivo lateral superior (12), e 1 canino superior (13), cinco pré-molares inferiores $(31,32,33$, $34,35,43,44$ e 45 , todos com canais únicos) que foram extraídos de um mesmo paciente, de 50 anos de idade, gênero masculino, de cor branca, que procurou por tratamento odontológico na clínica de Periodontia do Curso de Odontologia do UNIFEB. Os dentes foram extraídos por indicação periodontal e protética e doados por meio da assinatura do Termo de Consentimento Livre e Esclarecido.

Os dentes molares superiores foram mensurados em uma única sessão, e os demais, em uma sessão seguinte, com dois dias de intervalo. Em ambos os casos, a sequência operatória foi a mesma, apenas diferindo o tipo de anestesia, que foi infiltrativa e troncular, nos molares superiores, e infiltrativa nos demais. Inicialmente, o paciente foi submetido à anestesia local com Mepivacaína a 2\% (Nova DFL Produtos Odontológicos, RJ, Brasil). A seguir, o isolamento absoluto foi realizado e procedeu-se à higienização dos dentes, lençol de borracha, grampos e arco com uma gaze embebida em álcool $70 \%$ (UNIFEB, Barretos, SP, Brasil), para realizar a desinfecção superficial do campo operatório. As aberturas coronárias dos dentes foram realizadas com fresas esféricas diamantadas número 1014 (KG Sorensen, Cotia - SP - Brasil) e tronco-cônica 
carbide Endo Z (Dentsply, Maillefer, Suíça), para a remoção do teto da câmara pulpar e das suas reentrâncias. Em seguida, a câmara pulpar de cada dente foi irrigada de forma abundante com hipoclorito de sódio 0,5\% (UNIFEB, Barretos, SP, Brasil). Em imediato, as medidas odontométricas foram realizadas com os localizadores apicais Justy II (Yoshida Toei, Japão), Mini Root ZX (J. Morita, Japão), e Propex II (Dentsply, Maillefer, Suíça).

Para isto, uma lima tipo Kerr (Dentsply, Petrópolis, RJ, Brasil) número 10 (no caso de canais atrésicos) ou número 15 (no caso de canais mais amplos) foi acoplada ao conector do aparelho de localização apical e introduzida com suaves movimentos de vai e vem com as câmaras pulpares inundadas com solução de hipoclorito de sódio $0,5 \%$ até que o aparelho emitisse um sinal sonoro indicando que foi atingido o limite apical. Foi realizado o ajuste do top apical no ponto de referência previamente determinado, aferimento do comprimento da lima com uma régua milimetrada (Dentsply, Maillefer, Suíça), e descontado $1 \mathrm{~mm}$ do comprimento para obtenção do comprimento real de trabalho (Comprimento Real de Trabalho). Os comprimentos foram anotados em planilha própria.

Os dentes foram então extraídos e, imediatamente após, os canais radiculares foram mensurados com as limas tipo Kerr manuais, citadas previamente. A introdução da lima em cada dente foi realizada até que sua ponta pudesse ser visualizada no forame apical com o auxílio de uma lupa de aumento de 10 vezes. Do valor obtido, foi subtraído $1 \mathrm{~mm}$ do comprimento para obtenção do CRT.

A análise estatística foi desenvolvida por meio de um programa específico (BioEstat 5.0, Sociedade Civil Mamirauá / MCT - CNPq, Belém, Brasil), considerando a hipótese nula baseada na ausência de diferença entre os grupos $(\alpha=5 \%)$. Os dados experimentais foram analisados pelo teste de normalidade Shapiro-Wilk, e como apresentaram distribuição não normal, utilizou-se o teste Friedman para comparações entre os grupos.

\section{Resultados}

Dados de todos os dentes puderam ser incluídos na avaliação. Porém, no caso do dente 28 , apenas a raiz palatina foi avaliada, devido ao fato de a lima manual introduzida no interior dos canais mésio-vestibular e disto-vestibular não conseguir emergir nos forames apicais, sendo as medidas descartadas. Os valores obtidos no estudo são apresentados na Tabela 1.

$\mathrm{Na}$ análise estatística entre os grupos, observou-se que as medidas do aparelho Mini Root ZX (mediana de $0,0 \mathrm{~mm}$; intervalo interquartílico de $-0,5 \mathrm{a} 1 \mathrm{~mm}$ ) foram mais próximas da medida de referência do que as medidas do aparelho Propex II (mediana de 1,0 mm; intervalo interquartílico de 0,0 a $1 \mathrm{~mm})(\mathrm{p}=0,02$, teste Friedman).

Houve coincidência da medida de referência com a dos aparelhos Just II, Mini Root ZX e Propex II em respectivamente 26,1\% (seis raízes), 30,4\% (sete raízes) e $21,7 \%$ (5 raízes) das mensurações. Nos casos discordantes, observou-se uma tendência à subestimação dos resultados, visto que a maioria das medidas apresentara valor positivo (64\%, $62,5 \%$ e $88,9 \%$ ), respectivamente.

A maior amplitude de variação foi do aparelho Just II (4,0 mm, variando de $-1,5 \mathrm{~mm}$ a $2,5 \mathrm{~mm})$. A menor foi do Mini Root ZX (2,5 mm, variando de $-1,0 \mathrm{~mm}$ a $1,5 \mathrm{~mm}$ ). O aparelho Propex II teve valor intermediário $(3,5 \mathrm{~mm}$, variando de $-0,5 \mathrm{~mm}$ a $3,0 \mathrm{~mm}$ ).

\section{Discussão}

A técnica eletrônica tem sido estudada e aprimorada desde a metade do século XX. A partir dos primeiros estudos de Sunada em 1962, a técnica eletrônica apresentou um apreciável desenvolvimento, superando os problemas iniciais apresentados, principalmente no que tange à incapacidade de leitura em canais que contêm soluções irrigadoras condutoras de corrente elétrica.

Santana (2007) mostrou-se preocupada com a dificuldade técnica de um perfeito selamento biológico da constrição apical, fato esse que implicaria o aumento dos índices de insucesso do tratamento endodôntico. O autor cita o uso de um aparelho capaz de registrar a passagem de corrente elétrica entre dois eletrodos posicionados no canal radicular e na mucosa bucal, com a finalidade de indicar o limite apical de instrumentação.

Nos últimos anos, estudos avaliaram que o método eletrônico resultou em índices de acerto satisfatórios, indicando que os localizadores foraminais eletrônicos encontraram lugar de destaque no contexto da clínica odontológica, na redução do tempo de trabalho, assim como menor irradiação do paciente por raios X (REAL et al., 2006). 
Tabela 1. Valores (em milímetros) do comprimento de trabalho dos canais radiculares após medição com diferentes aparelhos localizadores foraminais in vivo e das medidas de referência feitas com lima manual in vitro após extração; além de suas variações $(\Delta)$.

\begin{tabular}{|c|c|c|c|c|c|c|c|c|}
\hline \multirow[b]{2}{*}{ Dente } & \multirow[b]{2}{*}{ Raiz } & \multirow{2}{*}{$\begin{array}{l}\text { Medida de } \\
\text { referência } \\
\text { In vitro }\end{array}$} & \multicolumn{6}{|c|}{ Medidas com aparelho localizadores foraminais in vivo } \\
\hline & & & Just II & $\begin{array}{c}\Delta \\
\text { Just II }\end{array}$ & $\begin{array}{c}\text { Mini root } \\
Z X X \\
\end{array}$ & $\begin{array}{c}\Delta \text { Mini } \\
\text { root } Z X\end{array}$ & Propex II & $\begin{array}{c}\Delta \text { Propex } \\
\text { II }\end{array}$ \\
\hline \multirow[t]{3}{*}{18} & MV & 25 & 24 & 1 & 24 & 1 & 24 & 1 \\
\hline & DV & 25 & 24 & 1 & 24 & 1 & 24 & 1 \\
\hline & $\mathrm{P}$ & 24 & 24 & 0 & 24 & 0 & 24 & 0 \\
\hline \multirow[t]{3}{*}{17} & MV & 24,5 & 23,5 & 1 & 23,5 & 1 & 22,5 & 2 \\
\hline & DV & 23,5 & 23,5 & 0 & 24 & $-0,5$ & 22,5 & 1 \\
\hline & $\mathrm{P}$ & 24 & 24,5 & $-0,5$ & 25 & -1 & 23,5 & 0,5 \\
\hline \multirow[t]{2}{*}{15} & V & 26 & 24,5 & 1,5 & 25 & 1 & 25 & 1 \\
\hline & $\mathrm{P}$ & 25 & 23 & 2 & 24 & 1 & 24 & 1 \\
\hline 13 & única & 31 & 29,5 & 1,5 & 30 & 1 & 29,5 & 1,5 \\
\hline 12 & única & 25 & 26,5 & $-1,5$ & 26,5 & $-1,5$ & 25,5 & $-0,5$ \\
\hline 25 & única & 27 & 27,5 & $-0,5$ & 27,5 & $-0,5$ & 27 & 0 \\
\hline \multirow[t]{3}{*}{26} & MV & 24 & 21,5 & 2,5 & 24 & 0 & 21 & 3 \\
\hline & DV & 24 & 22 & 2 & 24 & 0 & 21,5 & 2,5 \\
\hline & $\mathrm{P}$ & 25 & 25,5 & $-0,5$ & 25,5 & $-0,5$ & 24,5 & 0,5 \\
\hline 28 & $\mathrm{P}$ & 20 & 20,5 & $-0,5$ & 20,5 & $-0,5$ & 19 & 1 \\
\hline 35 & única & 24 & 23 & 1 & 23 & 1 & 23 & 1 \\
\hline 34 & única & 23 & 23 & 0 & 23 & 0 & 22,5 & 0,5 \\
\hline 33 & única & 28 & 27 & 1 & 27 & 1 & 27 & 1 \\
\hline 32 & única & 23 & 23 & 0 & 23 & 0 & 23 & 0 \\
\hline 31 & única & 20,5 & 21 & $-0,5$ & 21 & $-0,5$ & 21 & $-0,5$ \\
\hline 43 & única & 27,5 & 27 & 0,5 & 27 & 0,5 & 27 & 0,5 \\
\hline 44 & única & 23 & 23 & 0 & 23 & 0 & 23 & 0 \\
\hline 45 & única & 23 & 23 & 0 & 23 & 0 & 23 & 0 \\
\hline \multicolumn{2}{|c|}{ Mediana } & 24,0 & 23,5 & $\mathbf{0 , 0}$ & 24,0 & $\mathbf{0 , 0 *}$ & 23,5 & $1,0 *$ \\
\hline \multicolumn{2}{|c|}{$\begin{array}{c}\text { Intervalo } \\
\text { interquartílico }\end{array}$} & $\begin{array}{c}23,25 \mathrm{a} \\
25,00\end{array}$ & $\begin{array}{c}23,50 \mathrm{a} \\
25,00\end{array}$ & $\begin{array}{c}-0,25 \mathrm{a} \\
1,00\end{array}$ & $\begin{array}{c}23,00 \mathrm{a} \\
25,25\end{array}$ & $\begin{array}{c}-0,50 \mathrm{a} \\
1,00\end{array}$ & $\begin{array}{c}22,50 \mathrm{a} \\
24,75\end{array}$ & $\begin{array}{c}0,00 \mathrm{a} \\
1,00\end{array}$ \\
\hline
\end{tabular}

* Diferença estatisticamente significante entre os grupos ( $\mathrm{p}=0,02$, teste Friedman).

Os aparelhos fundamentam-se na detecção da diferença entre dois ou mais valores de frequência, para determinar a constrição apical. O circuito interno identifica a diferença entre os valores de frequência (valor relativo), mediante um ajuste inicial, independentemente das condições de umidade do canal, fornecendo um valor constante, equivalente à diferença entre as frequências mensuradas (VASCONCELOS et al., 2013).

À medida que a ponta da lima se posiciona perto do forame apical, determina-se uma variação desproporcional nos valores de impedância, medidos pelas frequências. A diferença entre as variações de impedância é detectada pelo aparelho, indicando o movimento no visor, antes posicionado na demarcação zero, referente a um valor inicial, que se mantinha constante até próximo ao início do terço apical. A interpretação desse movimento, aliada a um alarme sonoro, indica a posição aproximada da constrição e a saída do forame apical (SILVA e ALVES, 2011).

A leitura da diferença de potencial elétrico dos tecidos propicia a execução de medições eletrônicas. A parede dentinária do canal radicular exibe uma baixa condutividade elétrica, sendo que, à medida que se aproxima do terço apical, a camada de tecido dentinário torna-se menos espessa, diminuindo sua capacidade de isolamento elétrico (SILVA, 2012). Essa diminuição gradativa é interpretada, eletricamente, como diminuição da impedância da dentina. Os aparelhos baseados no método da frequência possuem uma calibragem que permite a indicação da variação de valores relativos de impedância da região apical, permitindo 
localizar a ponta do instrumento a cerca de $1 \mathrm{~mm}$ do forame apical, posição sugerida da constrição apical. Essa prerrogativa foi comprovada pelo estudo de Oishi et al., em 2002, quando avaliaram a capacidade do Root ZX de detectar a presença de constrições apicais em dentes com e sem patência foraminal.

As diferenças entre os resultados dos estudos comparando as técnicas eletrônicas e radiográficas foram detectadas devido ao fato de os aparelhos localizadores foraminais do tipo frequência indicarem a posição da constrição e saída do forame apical, enquanto que a análise radiográfica interpreta apenas a posição do ápice radiográfico, coincidente em menos de $61 \%$ dos casos com a posição real do forame apical (SCARPARO e NEUVALD, 2006).

O método da frequência apresentou valores, em média, precisos, quanto à localização do forame apical, sob condições clínicas de aferições estudadas na literatura endodôntica (CHITA et al., 2012). Por outro lado, Real et al. (2006), realizando análise comparativa da acurácia in vitro entre os localizadores apicais Just II e Root ZX, concluíram que os aparelhos não foram precisos na determinação do comprimento real de trabalho ( $1 \mathrm{~mm}$ aquém ápice).

Miguita et al. (2011), comparando in vitro os localizadores apicais Root ZX II e Propex na determinação do comprimento real de trabalho, observaram que houve diferenças estatísticas entre as medidas obtidas e recomendaram suas utilizações na clínica odontológica diária. Adicionalmente, Vasconcelos et al. (2013) avaliaram in vivo e in vitro os localizadores apicais Root $\mathrm{ZX}$ e o Propex II quanto à sua precisão na realização da odontometria durante o tratamento do canal radicular e observaram que o Root ZX apresentou maior confiabilidade, o que concorda com os achados do presente estudo, o qual mostrou que o Mini Root ZX apresentou tendência de melhor desempenho.

\section{Conclusões}

De acordo com o estudo realizado, pode-se concluir que:

1) Os três aparelhos testados demonstraram eficácia quanto à medida dos comprimentos de trabalho dos canais radiculares analisados.

2) $\mathrm{Na}$ comparação in vitro dos comprimentos reais dos canais radiculares dos dentes extraídos (experimento in vitro), não houve diferença estatística, quando comparadas às medidas odontométricas realizadas in vivo pelos localizadores apicais.

3) O aparelho Root ZX-II apresentou tendência de melhor desempenho que os demais.

\section{Referências}

BRITO JUNIOR, M.; CAMILO, C.C.; OLIVEIRA, A.M.; SOARES, J.A. Precisão e confiabilidade de um localizador apical na odontometria de molares inferiores, in vitro. Revista Odonto Ciência, v. 294, n. 8, p. 293-298, 2007.

CESÁRIO, F.; GUIMARÃES, B.M.; PINTO, L.C.; NISHIYAMA, C.K. Avaliação da precisão de três localizadores foraminais na determinação do comprimento de trabalho: um estudo in vitro. SALUSVITA, Bauru, v. 33, n. 2, p. 169-179, 2014.

CHITA, J.J.; SILVA, P.G.; PEREIRA, K.F.S.; ONODA, H.K.; BORBA JUNIOR, J.C.; RAMOS, C.A.S. Precisão e confiabilidade de um novo localizador foraminal eletrônico-Estudo in vivo. Pesquisa Brasileira em Odontopediatria e Clinica Integrada, v. 12, n. 4, p. 457-463, 2012.

FREITAS, F.; DANTAS, W.C.F.; CREPALDI, M.V.; BURGER, R.C. Localizadores apicais. Revista FAIPE, v. 2, n. 2, p. 44-66, 2012.

GORDON, M.P.J.; CHANDLER, N.P. Eletronic apex locators. International Endodontic Journal, v. 37, p. 425-437, 2004.

KUTTLER, Y. Microscopic investigation of root apexes. The Journal of the American Dental Association, v. 50, p. 544-552, 1955.

LUCISANO, M.P.; LEONARDO, M.R.; NELSON FILHO, P.; SILVA, R.A.B. Utilização de localizadores eletrônicos foraminais na determinação da odontometria em dentes decíduos. Ciência Odontológica Brasileira, v. 12, n. 2, p. 73-81, 2009.

LISKA, L.S. Avaliação in vitro da eficácia de dois localizadores apicais eletrônicos na determinação do comprimento real de trabalho do canal radicular [trabalho de conclusão de curso]. Rio Grande do Sul: UNINGÁ Passo Fundo, Faculdade de Odontologia INGÁ, 2009. 
MATTAR, R.; ALMEIDA, C.C. Análise da interferência em localizador apical eletrônico modelo Root ZX quando utilizado em dentes com reabsorção radicular simulada. A Revista Odontológica do Brasil-Central, v. 17, n. 43, p. 13-21, 2008.

MIGUITA, K.B.; CUNHA, R.S.; DAVINI, F.; FONTANA, C.E.; SILVEIRA, C.E. Análise comparativa de dois localizadores apicais eletrônicos na definição do comprimento de trabalho na terapia endodôntica: estudo in vitro. Revista Sul-Brasileira de Odontologia, v. 8, n. 1, p. 27-32, 2011.

OISHI, A.; YOSHIDA, T.; KOBAYASHI, C.; SUDA, H. Eletronic detection of root canal constrictions. Journal of Endodontics, v. 28, n. 5, p. 361-364, 2002.

OROSCO, F.A. Influência da espessura de dentina, da constrição apical e do diâmetro do forame apical na precisão de leitura com localizadores foraminais eletrônicos [tese]. São Paulo: Universidade de São Paulo, Faculdade de Odontologia de Bauru, 2010.

REAL, D.G.; CARVALHO, A.L.P.; PALEARI, G.S.L.; OKINO, K.; MOURA, A.A.M.; DAVIDOWISCH, H. Análise comparativa "in vitro" entre localizadores apicais eletrônicos Just II e Root ZX. Revista do Instituto de Ciências da Saúde, v. 24, n. 3, p. 201-205, 2006.

SANTANA, B.P. Avaliação ex vivo da precisão do localizador eletrônico Root ZX II na determinação da posição do limite CDC, utilizando-se o registro
1 do visor [dissertação]. Uberlândia: Universidade Federal de Uberlândia, Faculdade de Odontologia, 2007.

SCARPARO, R.K.; NEUVALD, L.R. Avaliação dos métodos radiográfico e eletrônico para a determinação do comprimento de trabalho em endodontia: estudo in vivo. Revista da Faculdade de Odontologia - UPF, v. 11, n. 2, p. 50-55, 2006.

SILVA, T.M.; ALVES, F.R.F. Localizadores apicais na determinação do comprimento de trabalho: a evolução através das gerações. Revista Brasileira de Odontologia, v. 68, n. 2, p. 180-185, 2011.

SILVA, T.M.S. Comparação ex vivo da precisão de três localizadores apicais na detecção do forame apical [dissertação]. Rio de Janeiro: Universidade Estácio de Sá, Faculdade de Odontologia, 2012.

SIU, C.; MARSHAL, G.; BAUMGARTNER, J. An in vitro comparison of the Root ZX II, the apex, and mini apex locator by using rotary nickel titanium files. Journal of Endodontics, v. 35, p. 962-965, 2009.

SUNADA, I. New method for measuring the length of the root canal. Journal of Dental Research, v. 41, p. 375-387, 1962.

VASCONCELOS, B.C.; BUENO, M.M.; LUNA CRUZ, S.M.; DUARTE, M.A.H.; FERNANDES, C.A.O. Accuracy of five electronic foramen locators with different operating systems: an ex vivo study. Journal of Applied Oral Science, v. 21, n. 2, p. 132-137, 2013. 